\title{
Aplicação da escala reduzida de autoeficácia em amamentação no contexto da Estratégia Saúde da Família
}

Recebido em: 03/10/2012

Aprovado em: 16/05/2014

\author{
Lorena Sousa Soares ${ }^{1}$ \\ Grazielle Roberta Freitas Da Silva² \\ Márcia Teles de Oliveira Gouveia ${ }^{3}$ \\ Erlayne Camapum Brandão ${ }^{4}$ \\ Mônica Oliveira Batista Oriás
}

Resumo: O objetivo do estudo foi avaliar a auto-eficácia em amamentação por meio da aplicação da Breastfeeding Self-Efficacy Scale - Short Form, junto a 39 mães, na cidade de Teresina (PI). Resultados mostraram eficácia alta e média, não existindo assim, eficácia baixa neste estudo. Na análise separada dos itens, os menores índices foram nos itens: 6 e 13, quanto à categoria técnica. No domínio intrapessoal, os menores índices foram nos itens: 2, 8 e 10. Identificando as mães que tem menor auto-eficácia e maior risco para o desmame precoce, o profissional pode avaliar e programar a sua promoção ao aleitamento materno.

Descritores: Aleitamento Materno, Validade dos Testes, Enfermagem em Saúde Comunitária.

\section{Reduced scale application of self-efficacy in breast feeding in the context of family health strategy}

Abstract: The purpose of this study was to evaluate self-efficacy in breastfeeding through the application of Breastfeeding Self-Efficacy Scale - Short Form, along with 39 mothers, in the city of Teresina (PI). Results showed average and high efficiency, so there is, low efficiency in this study. In separate analysis of scale items, the lowest levels were the following items: 6 and 13 about the technical category. In the intrapersonal domain, the lowest rates were in the items: 2,8 and 10 . Identifying mothers who have lower self-efficacy and higher risk for early weaning, the practitioner can assess and plan its promotion of breastfeeding.

Descriptors: Breast Feeding, Validity of Tests, Community Health Nursing.

Reuccion de aplicación a gran escala de autoeficacia en la lactaricia materna en el contexto de la estrategia de salud de la família.

Resumen: El propósito de este estudio fue evaluar la autoeficacia en la lactancia materna a través de la aplicación de Breastfeeding Self-Efficacy Scale Short Form, entre 39 madres de la ciudad de Teresina (PI). Los resultados mostraron una alta eficacia y la media, por lo que es, una baja eficacia en este estudio. En un análisis separado de los elementos, el más bajo en los artículos fueron: 6 y 13, como la categoría de técnico. En el dominio intrapersonal, las tasas más bajas en los artículos: 2,8 y 10. La identificación de madres que tienen menor riesgo de auto-eficacia y el aumento de destete precoz, el médico puede evaluar y planificar la promoción de la lactancia.

Descriptores: Lactancia materna, Validez de las pruebas, Enfermería en Salud Comunitaria.

\section{INTRODUÇÃO}

$\mathrm{O}$ aleitamento materno não é a simples ação de colocar a criança ao peito da mãe, é um processo complexo que envolve experiências culturais, sociais, psíquicas e biológicas da mulher. Como uma tarefa há ser cumprida em prol da saúde da criança, existe uma cobrança social para a mãe amamentar, porém vários fatores dificultam ou inviabilizam esta prática.

Apesar dos conhecimentos atuais confirmarem a importância do aleitamento materno exclusivo (AME) para crianças até os seis meses de idade, a introdução precoce de líquidos e alimentos artificiais em crianças amamentadas continua sendo freqüente em nosso meio. Tal fato pode ser evidenciado quando $32,7 \%$ das mães julgaram como incorreta a assertiva "outros alimentos podem causar substituição do AME", pois acreditam que a inserção de outros alimentos não influencia na duração do $\mathrm{AME}^{(1)}$.

Na assistência de enfermagem, na promoção da amamentação, pela interação profissional e mãe, pode-se desenvolver um plano de cuidados possível de ser realizado em todos os níveis de atenção, pois a partir deste compartilhamento de pensamentos e valores, 0 enfermeiro é capaz de conhecer as reais necessidades do binômio mãe-filho, para poder, assim, intervir.

No Brasil, as estimativas das prevalências do aleitamento materno mostram que a maioria das crianças $(87,3 \%)$ é amamentada no primeiro mês de vida. Essa proporção decresce para $77,5 \%$ aos 120 dias, e para 68,6\%, aos 180 dias. Na cidade de Teresina (PI), estes percentuais são $92,7 \% ; 86,4 \%$ e $80,1 \%$, respectivamente. Quanto aos valores do aleitamento materno exclusivo, as taxas são ainda menores. Os percentuais na idade de 30, 120 e 180 dias, em Teresina, caem para 55,0\%; 22,0\% e 9,6\%, respectivamente ${ }^{(2)}$. Estas taxas deixam claro que apesar das várias estratégias de incentivo ao AME, elas ocorrem isoladamente até contrapõem-se, o que demonstra a falta de articulação das ações de saúde.

No que se refere à atuação dos serviços de saúde, há três pontos em que estimular e favorecer as condições para o aleitamento podem fazer diferença e, inclusive, influenciar o aleitamento materno exclusivo: incentivo e apoio nas consultas de pré-natal; promoção do contato na maternidade imediatamente após o parto e continuação do apoio nas primeiras consultas do recém-nascido ${ }^{(3)}$.

'Enfermeira. Aluna do Curso de Mestrado em Enfermagem pela Universidade Federal do Piauí (UFPI). E-mail: Iorenacacaux@hotmail.com

${ }^{2}$ Enfermeira. Doutora em enfermagem. Docente do Programa de pós-graduação em enfermagem da UFPI.

${ }^{3}$ Enfermeira. Mestre em Saúde da Criança. Docente da UFPI.

${ }^{4}$ Enfermeira. Aluna do Curso de Mestrado em Enfermagem pela Universidade de Brasília (UnB).

${ }^{5}$ Enfermeira. Doutora em enfermagem. Docente do Programa de pós-graduação em enfermagem da Universidade Federal do Ceará (UFC). 
O estudo tem por objetivo avaliar a autoeficácia em amamentação por meio do escore obtido na aplicação da Breastfeeding Self-Efficacy Scale - Short Form (BSES-SF).

\section{METODOLOGIA}

Estudo descritivo, transversal e com abordagem quantitativa, realizado em uma Unidade Básica de Saúde (UBS) que engloba sete equipes da Estratégia Saúde da Família localizada na cidade de Teresina (PI). A população foi composta por 39 mães que amamentavam e que compareceram ao serviço para consulta de puericultura na referida UBS, durante os meses pré-estabelecidos para a coleta de dados.

A coleta de dados foi realizada nos meses de novembro de 2010 a março de 2011, no turno da manhã, às segundas e terças-feiras, dias destinados, de acordo com a rotina, para realização das consultas de puericultura da referida instituição. Antes ou após a consulta de puericultura realizada pelo enfermeiro, aplicou-se a BSES-SF(4).

A BSES-SF é uma escala estruturada composta de 14 itens que abordam duas categorias de domínio: técnica e pensamentos intrapessoais. Cada item é avaliado de acordo com uma escala de concordância (tipo Likert) com a seguinte pontuação: 1-discordo totalmente, 2-discordo, 3-às vezes concordo, 4-concordo e 5-concordo totalmente ${ }^{(5)}$. Para facilitar a interpretação das mães, foi desenvolvido um instrumento, correlacionando as respostas às cores respectivas: 1. discordo totalmente (vermelho), 2. discordo (laranja), 3. às vezes concordo (amarelo), 4. concordo (azul) e 5. concordo totalmente (verde), o que facilitou o entendimento das mães em relação às respostas de cada questão.

Um banco de dados em planilha eletrônica foi elaborado, em programa Excel 2007, para procedimento das análises descritivas e inferenciais e, as informações coletadas foram exportadas e organizadas no Programa Statistical Package for the Social Sciences (SPSS) versão 17.0.

O estudo, como parte integrante do Programa de Bolsa de Iniciação Científica (PIBIC), foi encaminhado e aprovado pelo Comitê de Ética da UFPI com o CAAE 0075.0.045.000-10.

\section{RESULTADOS E DISCUSSÃO}

A escala total, com 14 itens, cada um com a pontuação variando de 1 a 5 pontos, apresenta, portanto, um mínimo de 14 e um máximo de 70 pontos. Com a finalidade de se analisar a eficácia, ela foi transformada da seguinte maneira(6): eficácia baixa (de 14 a 32 pontos), eficácia média (de 33 a 51 pontos) e eficácia alta (de 52 a 70 pontos).

A eficácia da amostra estudada variou de 51 a 70 pontos, ou seja, duas obtiveram eficácia média e 37, eficácia alta, não existindo assim, eficácia baixa neste estudo.

Tabela 1 - Distribuição das mães segundo os itens do domínio Técnico da BSFS - SF. Teresina, PI, 2011.

\begin{tabular}{cccccccccccc}
$\begin{array}{c}\text { Domínio } \\
\text { Técnico }\end{array}$ & $\begin{array}{c}\text { Concordo } \\
\text { totalmente }\end{array}$ & \multicolumn{2}{c}{ Concordo } & $\begin{array}{c}\text { As vezes } \\
\text { concordo }\end{array}$ & Discordo & $\begin{array}{c}\text { Discordo } \\
\text { totalmente }\end{array}$ \\
Item & $\mathbf{n}$ & $\%$ & $\mathbf{n}$ & $\%$ & $\mathbf{n}$ & $\%$ & $\mathbf{n}$ & $\%$ & $\mathbf{n}$ & $\%$ \\
\hline $1(\mathrm{n}=39)$ & 15 & 38,5 & 16 & 41,0 & 8 & 20,5 & - & - & - & - \\
\hline 3 & 15 & 38,5 & 16 & 41,0 & $8 \%$ & 20,5 & - & - & - & - \\
\hline 4 & 22 & 56,4 & 17 & 43,6 & - & - & - & - & - & - \\
\hline 6 & 27 & 69,2 & 8 & 20,5 & 1 & 2,6 & 2 & 5,1 & 1 & 2,6 \\
11 & 21 & 53,8 & 14 & 35,9 & 2 & 5,1 & 1 & 2,6 & 1 & 2,6 \\
\hline 12 & 25 & 64,1 & 12 & 30,8 & 2 & 5,1 & - & - & - & - \\
13 & 17 & 43,6 & 13 & 33,4 & 6 & 15,4 & 2 & 5,1 & 1 & 2,6 \\
14 & 20 & 51,3 & 14 & 35,9 & 4 & 10,2 & 1 & 2,6 & - & - \\
\hline
\end{tabular}

Na categoria Domínio Técnico, a escala focaliza os aspectos técnicos do aleitamento, relacionados diretamente a execução do ato de amamentar, como a pega, a posição, a ordenha, entre outros (Tabela 1).

$\mathrm{Na}$ análise destes itens, a maior proporção entre as repostas do tipo"discordo e discordo totalmente" aconteceu nos seguintes: item 6 (Eu sempre posso amamentar mesmo se o meu bebê estiver chorando) e item 13 (Eu sempre consigo adequar as minhas necessidades às necessidades do bebê).

Assim, o fato de a proporção destes itens serem semelhantes, parece coerente, tendo em vista que é necessária certa técnica para amamentar e cuidar corretamente do bebê, assim, organizando suas necessidades de banho e sono com a amamentação ${ }^{(6)}$. Esta análise evidencia que as mães possuíam menor auto-eficácia nestes itens. Desta maneira, os profissionais de saúde, especificamente, os enfermeiros, devem enfatizar e esclarecer, nos momentos de orientação e aconselhamento em amamentação, os cuidados dispensados e essenciais para a saúde infantil, considerando o manejo técnico e as necessidades pessoais de cada mãe.

Na vivência cotidiana do profissional de saúde, ações verticalizadas e normatizadas, bem como o reducionismo biológico, se fazem presentes, negligenciando outras facetas inerentes ao processo saúde-doença, tais como os aspectos histórico, social e cultural. Para os profissionais de saúde, o significado da amamentação aparece pautado na possibilidade de a mãe dar o melhor para o bebê, ou seja, uma condição emblemática de ser uma boa mãe. De nada adianta um profissional consciente da importância da amamentação e atuante em sua promoção se o mesmo fornecer informações erradas às mães ou se não souber apoiá-las em manejos adequados ${ }^{(7)}$.

As principais atividades práticas que representam oportunidades de orientação, apoio e incentivo ao aleitamento materno são: orientação desde a fase inicial do pré-natal; orientação ao final do pré-natal; estimulação da amamentação nas atividades de puericultura; avaliação da mamada durante as visitas; orientação para prevenção de problemas com as mamas e realização de visitas puerperais precocemente ${ }^{(8)}$.

Houve um nível alto de concordância das mães nos itens 1 (Eu sempre sinto quando o meu bebê está mamando o suficiente), 3 (Eu sempre alimento o meu bebê sem usar leite em pó como suplemento), 4 (Eu sempre percebo se o meu bebê está pegando o peito direitinho durante toda a mamada) e 12 (Eu sempre continuo amamentando meu bebê a cada alimentação dele), que apresentaram valores maiores para respostas do tipo "às vezes concordo, concordo e concordo totalmente", além disso o item 4 foi o único com respostas apenas do tipo "concordo e concordo totalmente". Este achado evidencia que as mães possuíam maior auto-eficácia nestes itens, especialmente, na percepção da correta pega durante a mamada. 
Tabela 2 - Distribuição das mães segundo os itens do domínio Pensamentos Intrapessoais da BSFS - SF. Teresina, PI, 2011.

\begin{tabular}{ccccccccccc}
$\begin{array}{c}\text { Domínio } \\
\text { Pensamentos } \\
\text { Intrapessoais }\end{array}$ & $\begin{array}{c}\text { Concordo } \\
\text { totalmente }\end{array}$ & Concordo & $\begin{array}{c}\text { Às vezes } \\
\text { concordo }\end{array}$ & Discordo & $\begin{array}{c}\text { Discordo } \\
\text { totalmente }\end{array}$ \\
Item & $\mathbf{n}$ & $\%$ & $\mathbf{n}$ & $\%$ & $\mathbf{n}$ & $\%$ & $\mathbf{n}$ & $\%$ & $\mathbf{n}$ & $\%$ \\
\hline $2(n=39)$ & 20 & 51,3 & 14 & 35,9 & 2 & 5,1 & 1 & 2,6 & 2 & 5,1 \\
\hline 5 & 24 & 61,5 & 15 & 38,5 & - & - & - & - & - & - \\
\hline 7 & 24 & 61,5 & 13 & 33,4 & 2 & 5,1 & - & - & - & - \\
\hline 8 & 26 & 66,7 & 10 & 25,6 & 2 & 5,1 & - & - & 1 & 2,6 \\
\hline 9 & 25 & 64,1 & 14 & 35,9 & - & - & - & - & - & - \\
\hline 10 & 21 & 53,8 & 15 & 38,5 & 2 & 5,1 & 1 & 2,6 & - & - \\
\hline
\end{tabular}

Na categoria Pensamentos Intrapessoais, a escala considera o desejo de amamentar, a motivação interna para a amamentação, a satisfação com a experiência de amamentar, dentre outros fatores relacionados à interação e à subjetividade materna.

$\mathrm{Na}$ análise destes itens, a maior proporção entre as repostas do tipo "discordo e discordo totalmente" aconteceu nos seguintes: item 2 (Eu sempre lido com amamentação com sucesso, da mesma forma que eu lido com outros desafios); item 8 (Eu posso dar de mamar confortavelmente na frente de pessoas da minha família) e item 10 (Eu sempre posso lidar com o fato de que amamentar exige tempo). Esta análise mostrou que as mães possuem menor auto-eficácia nestes itens.

Além da maior proporção entre as repostas do tipo"discordo e discordo totalmente" ter sido evidente em dois itens do domínio Técnico, no de Pensamentos Intrapessoais houve também este mesmo achado em três itens, mostrando assim, a deficiência e a baixa qualidade das orientações fornecidas, não apenas no âmbito técnico, como também, na comunicação, na interação e na relação subjetiva entre a mãe e o profissional de saúde. Inúmeros fatores, já citados anteriormente, podem influenciar, prejudicar, ou até mesmo, impossibilitar o repasse de informações positivas sobre a amamentação e o incentivo do aleitamento exclusivo.

A dificuldade para amamentar nos primeiros dias mostra-se fortemente associada ao desmame, independente de outros fatores. É atribuição do profissional de saúde prestador da assistência a mulheres e crianças promover o aleitamento materno, na sua forma ampliada, através de ações que tenham por objetivo a sensibilização, a promoção, o incentivo e o apoio a esta prática.
Por isso, é fundamental que o enfermeiro não cuide somente com abordagem técnica, mas que sua perspectiva seja ampliada, através de uma assistência associada aos aspectos socioculturais da amamentação ${ }^{(9)}$.

Os itens 5 (Eu sempre lido com a amamentação de forma a me satisfazer), 7 (Eu sempre sinto vontade de continuar amamentando)e 9 (Eu sempre fico satisfeita com a minha experiência de amamentar) apresentaram valores maiores para respostas do tipo "às vezes concordo, concordo e concordo totalmente", além disso, os itens 5 e 9 foram os únicos com respostas apenas do tipo "concordo e concordo totalmente". Isto mostrou que as mães possuíam maior auto-eficácia nestes itens, principalmente, na satisfação em amamentar e na perspectiva de continuar amamentando seus bebês, apesar das dificuldades de adaptação do próprio cotidiano.

Entretanto, em outro estudo(4), verificou-se um maior percentual de discordância (discordo e discordo totalmente) no item 5. Tal fato demonstra que a percepção das mães está mudando, pois há décadas, o aleitamento foi veiculado como essencial para a criança, desconsiderando a importância de decisão e os anseios das mulheres. Assim, as mães, atualmente, estão começando a acreditar e consolidar que a amamentação deve satisfazê-las também, e que, elas são a principal figura deste processo.

\section{CONCLUSÃO}

A partir da aplicação da BSES - SF em mães que amamentavam, inseridas no contexto da Estratégia Saúde da Família, e da análise dos escores obtidos, concluiu-se que esta escala é um instrumento de fácil aplicação, principalmente com a adaptação da tabela de cores, facilitando assim, a compreensão das perguntas e a correlação das cores com as respostas. Esse último aspecto foi muito importante, o que remete aos autores da escala a sua implementação.

A BSES - SF é um método confiável, para avaliar a percepção, a auto-eficácia e a promoção do aleitamento materno, podendo, assim, ser relevante na rotina de trabalho dos enfermeiros que atendem na ESF, especialmente, nas consultas de pré-natal e puericultura e nas visitas domiciliares de puérperas, pois a escala é um instrumento que mede e diagnostica a confiança das mães no aleitamento materno e no seu potencial para amamentar. Identificando as mães que tem menor auto-eficácia e, conseqüentemente, maior risco para o desmame precoce, o profissional pode avaliar e programar a sua promoção ao AM, por intervenções individualizadas e orientações que promovam a saúde e o bem-estar do bebê, da mãe e de toda a família.

\section{Referências}

1. Silva VMM, Joventino ES, Silva SF, Martins MC, Vera

JEGLF, Ximenes LB. Conhecimento das mães de neonatos

sobre aleitamento materno. ReTEP - Revista Tendências da

Enfermagem Profissional. 2009; 1(1): 7-12.

2. Sena MCF, Silva EF, Pereira MG. Prevalência do aleitamento materno nas capitais brasileiras. Rev. Assoc. Med. Bras. [online]. 2007; 53(6): 520-524.

3. Dodt RCM, Oriá MOB, Silva MJ, Braga VAV, Ximenes LB. Políticas públicas e aleitamento materno. ReTEP - Revista Tendências da Enfermagem Profissional. 2010; 2(2): 42-48. 4. Dodt RCM. Aplicação e validação da Breastfeeding SelfEfficacy Scale - Short Form (BSES - SF) em puérperas. [tese de doutorado]. Fortaleza: Universidade Federal do Ceará; 2008.

Disponível em: http://www.objnursing.uff.br/index.php/nursing/ article/view/j.1676-4285.2010.2717/html_79.

6. Oriá MOB. Tradução, adaptação e validação da Breastfeeding SelfEfficacy Scale: aplicação em gestantes. [dissertação de mestrado]. Fortaleza: Universidade Federal do Ceará; 2008.

7. Marques ES, Cotta RMM, Franceschin SCC, Botelho MIV, Araújo RMA. Práticas e percepções acerca do aleitamento materno: consensos e dissensos no cotidiano de cuidado numa Unidade de Saúde da Família. Physis [online]. 2009; 19(2): 439-455.

8. Caldeira AP, Aguiar GN, Magalhães WAC, Fagundes GC. Conhecimentos e práticas de promoção do aleitamento materno em Equipes de Saúde da Família em Montes Claros, Minas Gerais, Brasil. Cad. Saúde Pública. 2007; 23(8): 1965-1970.

9. Almeida CC, Scochi MJ, Souza RKT, Carvalho WO. Prevalência de aleitamento materno antes e após a implantação de um programa de redução de morbimortalidade infantil, no município de Campo Mourão (PR): revisão. Ciênc. saúde coletiva. 2010; 15(2): 575-580. 